\title{
Heritable temperament pathways to early callous-unemotional behaviour
}

Rebecca Waller, Christopher J. Trentacosta, Daniel S. Shaw, Jenae M. Neiderhiser, Jody M. Ganiban, David Reiss, Leslie D. Leve and Luke W. Hyde

\section{Background}

Early callous-unemotional behaviours identify children at risk for antisocial behaviour. Recent work suggests that the high heritability of callous-unemotional behaviours is qualified by interactions with positive parenting.

\begin{abstract}
Aims
To examine whether heritable temperament dimensions of fearlessness and low affiliative behaviour are associated with early callous-unemotional behaviours and whether parenting moderates these associations.

\section{Method}

Using an adoption sample $(n=561)$, we examined pathways from biological mother self-reported fearlessness and affiliative behaviour to child callous-unemotional behaviours via observed child fearlessness and affiliative behaviour, and whether adoptive parent observed positive parenting moderated pathways.
\end{abstract}

\section{Results}

Biological mother fearlessness predicted child callousunemotional behaviours via earlier child fearlessness. Biological mother low affiliative behaviour predicted child callous-unemotional behaviours, although not via child affiliative behaviours. Adoptive mother positive parenting moderated the fearlessness to callous-unemotional behaviour pathway.

\section{Conclusions}

Heritable fearlessness and low interpersonal affiliation traits contribute to the development of callous-unemotional behaviours. Positive parenting can buffer these risky pathways.

\section{Declaration of interest}

None.

\section{Copyright and usage}

(C) The Royal College of Psychiatrists 2016.
Antisocial behaviour, including violence, theft, and substance use, is a major public health concern because of its striking economic, social, and health effects on perpetrators, victims, and families. Moreover, antisocial behaviour places a substantial financial burden on society through demands on health and education services, and incarceration costs. ${ }^{1}$ Children with persistent behaviour problems, starting in the toddler period, are at risk for developing severe antisocial behaviour that can lead to incarceration. ${ }^{2}$ Interventions initiated in early childhood when behaviour is more malleable can be efficacious for modifying costly, chronic trajectories of antisocial behaviour. ${ }^{3}$ However, the effectiveness of interventions is undermined because of heterogeneity in the developmental pathways leading to behaviour problems. ${ }^{4,5}$ By identifying different subgroups of children whose behaviour problems emerge from distinct aetiologies, it may be possible to develop more effective, personalised interventions.

\section{Callous-unemotional behaviours}

One approach for identifying a subgroup of children with severe behaviour problems is via the presence or absence of callousunemotional behaviours. Measures of callous-unemotional behaviours were created as a downward extension of the interpersonal/affective components of psychopathy, a complex set of personality traits that predicts violent and non-violent antisocial conduct among adults. ${ }^{6-8}$ In children, measures of callousunemotional behaviours assess empathy and guilt deficits, as well as reduced emotional responsivity to others' feelings or threat cues. $^{9}$ In late-childhood and adolescence, callous-unemotional behaviours robustly predict aggression, violence, and substance use, and are a risk factor for adult psychopathy and antisocial behaviour (see Frick et al $;^{9}$ Fig. 1). However, most research in this area has focused on samples in late-childhood and adolescence. Because early childhood is a period when behaviour is thought to be more malleable and preventative interventions may be more successful, ${ }^{3}$ we have recently begun to examine callousunemotional behaviours in the toddler and preschool periods. ${ }^{10}$ Note that our use of callous-unemotional 'behaviours' is consistent with prior work during this early childhood period (see Waller et $a l^{10-12}$ ). However, in older samples of children and adolescents, studies refer to callous-unemotional 'traits'. Given that there is little evidence to suggest that callousunemotional behaviours in early childhood and callousunemotional traits in late-childhood or adolescence are more than modestly-moderately stable, we prefer the term 'behaviours' because it has fewer stigmatising connotations of stability or lack of amenability to treatment that might reflect the origins of this construct in the adult psychopathy literature. Moreover, at this age, measurement relies on parent reports of observable child behaviours. Similar to findings from studies of older children, callous-unemotional behaviours measured in young children as early as age 3 are related to lower guilt and empathy, ${ }^{11}$ predict more proactive aggression and antisocial behaviours, ${ }^{1,14}$ and are uniquely related to callous-unemotional behaviours in late childhood $^{10}$ (Fig. 1). However, although callous-unemotional behaviours in early childhood foreshadow behaviour problems and callous-unemotional behaviours later in childhood, we know little about the developmental origins of callous-unemotional behaviours, and particularly temperament dimensions.

\section{Early temperament factors that may lead to callous-unemotional behaviours}

Temperament is defined as an enduring part of character influenced by heredity, biology, experience, and maturation. ${ }^{15}$ By studying temperament in infancy, we can identify early markers that might precede callous-unemotional behaviours and inform our understanding of developmental pathways from 


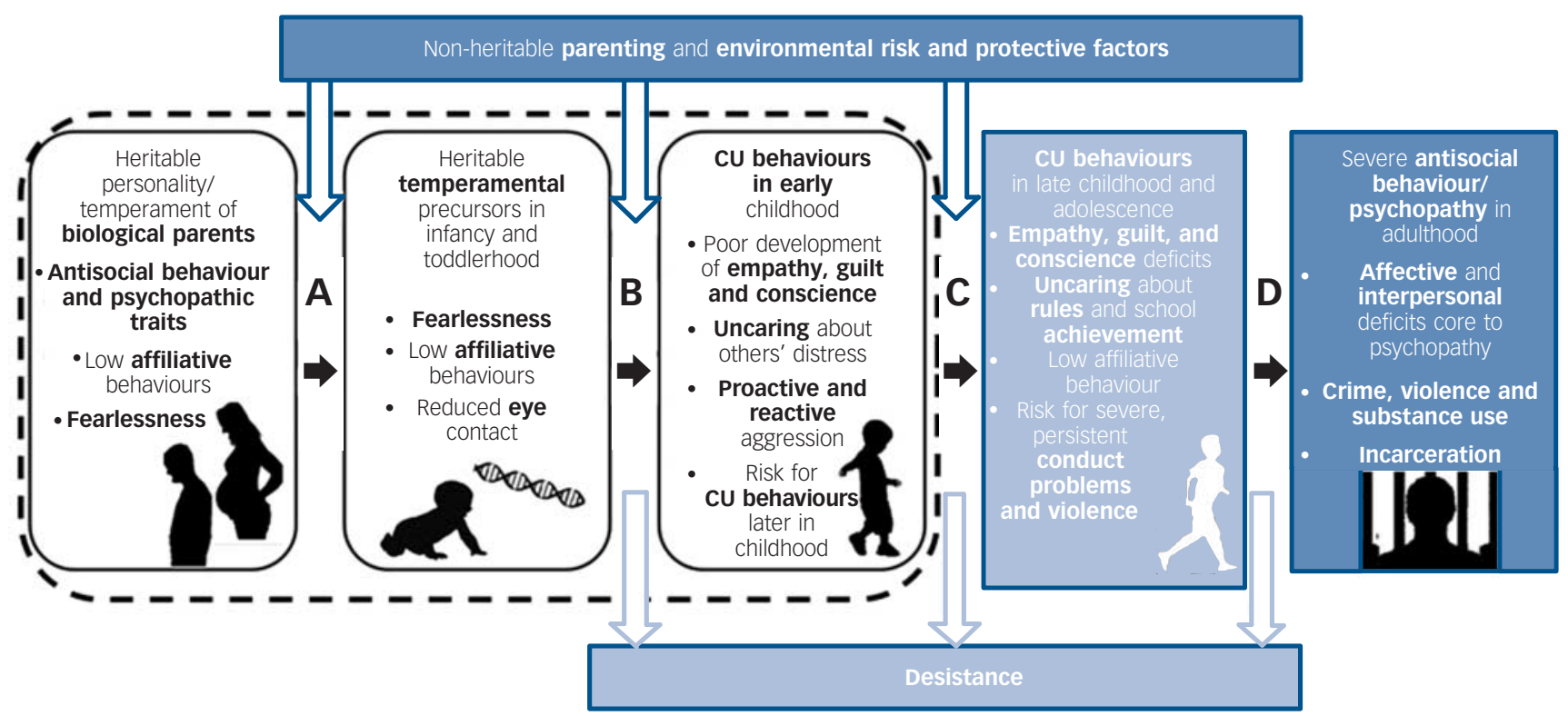

Fig. 1 Lifespan developmental model depicting a heritable callous-unemotional pathway to severe antisocial behaviour and psychopathy. We propose a pathway to antisocial behaviour and psychopathy originating with inherited temperament (A). Fearlessness and low affiliative behaviour in infancy are theorised to increase risk for early childhood callous-unemotional (CU) behaviours (B), which in turn predict behaviour problems and callous-unemotional behaviours across childhood and adolescence (C). Callous-unemotional behaviours increase the risk for severe forms of violence, aggression and psychopathic traits into adulthood (D). The current study tests pathways A and B, and examines the protective effects of positive parenting. At each point of the model, however, we propose moderating effects of the environment, whereby positive parenting and protective, environmental experiences reduce callous-unemotional behaviours (see reviews of parenting influences on callous-unemotional behaviours for pathways B and C of the model $l^{9,10,13}$ ).

callous-unemotional behaviours to antisocial behaviour and psychopathy (Fig. 1). Drawing on its origins in the psychopathy literature, callous-unemotionality is hypothesised to emerge from a 'fearless' temperament. Fearlessness is central to developmental models of psychopathy ${ }^{7}$ and conscience, ${ }^{16}$ as it is thought to confer low arousal to others' distress and punishment, leading to reduced learning about the outcomes of harmful behaviour, and increasing risk for callous-unemotional behaviours. Although it is prominent in theory, only two longitudinal studies have linked early fearlessness to psychopathy in adulthood ${ }^{17}$ or callous-unemotional behaviours in adolescence, ${ }^{18}$ and prospective research is needed to examine fearlessness in relation to callousunemotional behaviours during early childhood.

A second temperament dimension central to psychopathy is low affiliative behaviour, operationalised as low interpersonal warmth or affection. ${ }^{6}$ Although direct prospective links from low affiliative behaviour have not been tested, recent studies suggest that callous-unemotional behaviours are uniquely related to lower quality of positive affective parent-child interactions, including lower eye contact and warmth, ${ }^{12,19}$ as well as lower empathy. ${ }^{11}$ Affiliative behaviour is conceptualised as a basic dimension of temperament present from birth that guides children's non-cognitive responses within relational contexts, ${ }^{20}$ including emotional facial gestures, affective imitation, and mutually responsive orientation. ${ }^{19,20}$ In interaction with other dimensions of temperament, these responses facilitate a close, warm, and cooperative parent-child relationship, setting the stage for the development of empathy. ${ }^{15,20}$ A lack of mutually responsive orientation or reduced parent-child warmth evoked by low child affiliation during early childhood may undermine the parent-child relationship and evoke harsher parenting, increasing risk for callous-unemotional behaviour. ${ }^{12,15,19}$ Based on their potential importance to the development of callousunemotional behaviours, our first aim was thus to examine whether the temperament dimensions of fearlessness and low affiliative behaviour preceded the emergence of callousunemotional behaviours. We focused on early childhood to identify these temperaments before individual differences in callous-unemotional behaviours or related constructs, such as conscience and empathy, have fully emerged (Fig. 1).

\section{Heritable pathways to callous-unemotional behaviour}

Early temperament is thought to be moderately heritable and to define person $\times$ context interactions that affect later behaviours and personality. ${ }^{15}$ Studies have also shown that callous-unemotional behaviours in middle childhood are highly heritable. ${ }^{5}$ To examine heritable pathways to early callous-unemotional behaviours, we recently demonstrated that biological parent antisocial behaviour predicted child callousunemotional behaviours at 27 months within an adoption design that allowed for parsing of heritable and non-heritable pathways. ${ }^{21}$ However, this prior study did not isolate specific personality traits of biological parents that predicted temperament precursors to callous-unemotional behaviours. Thus, our second goal was to examine heritable temperament pathways in which biological parent fearlessness and low affiliative behaviour predicted child callous-unemotional behaviours via their influence on earlier child fearlessness and low affiliative behaviour. We examined pathways using an adoption study in which children were not raised by biological parents, thus excluding the possibility that heritable pathways actually resulted from parent-child interactions or other non-heritable pathways (Fig. 2).

\section{Parenting as a moderator of heritable risk}

Temperament pathways are likely to be modified by context, particularly parenting. ${ }^{16}$ In our previous work, the pathway from 


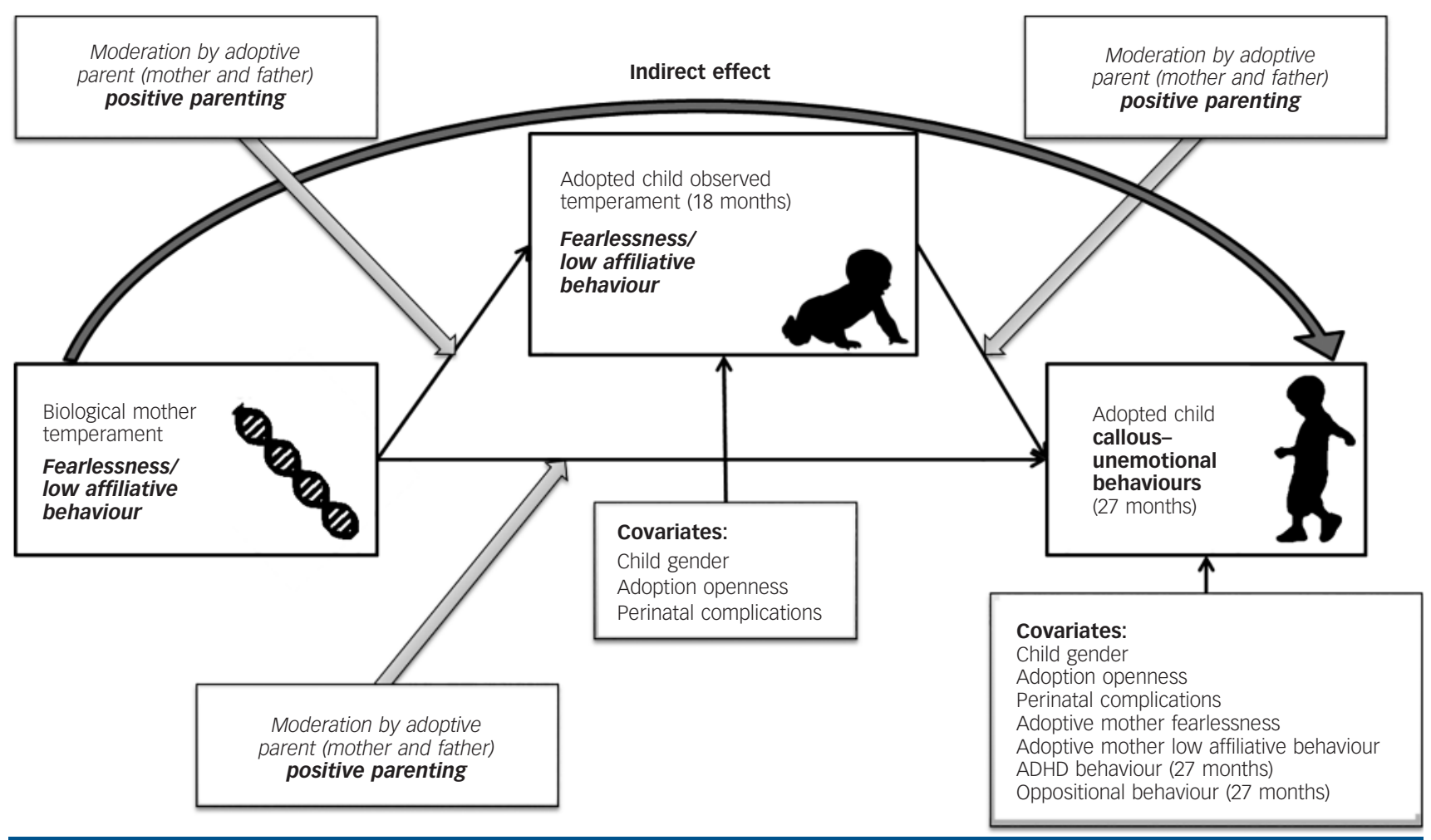

Fig. 2 Heritable pathways to early callous-unemotional behaviour via fearlessness and low affiliative behaviour buffered by non-heritable positive reinforcement of adoptive mothers.

We examined direct pathways from biological parent fearlessness and low affiliative behaviour to adopted child temperament at 18 months and callous-unemotional behaviours at 27 months, as well as direct paths from adopted child observed temperament to callous-unemotional behaviours. We tested an indirect pathway from biological parent temperament to adopted child callous-unemotional behaviours via observed child temperament. ADHD, attention-deficit hyperactivity disorder.

biological parent antisocial behaviour to child callousunemotional behaviours was moderated by observed positive parenting of adoptive mothers. ${ }^{21}$ Consistent with a growing literature showing that positive parenting - including warmth, responsivity, and praise - predict callous-unemotional behaviours across childhood, ${ }^{13,22}$ this interaction emphasises the malleability of heritable pathways. Moreover, this type of gene-environment interaction suggests that parent-child temperament pathways could also be moderated by caregiving quality. However, studies have yet to test whether positive parenting attenuates heritable temperament pathways. Thus, our third goal was to test whether adoptive parent positive parenting moderated heritable fearlessness and low affiliative behaviour pathways to callousunemotional behaviours. For both pathways, we focused on moderation effects from biological parent to child temperament (i.e. does positive parenting buffer heritable influences on early temperament?) and from child temperament to callousunemotional behaviours (i.e. does positive parenting buffer transitions from risky temperament to early callous-unemotional behaviours?).

\section{Current study}

We tested heritable temperament pathways to callousunemotional behaviours in a sample of 561 children from the Early Growth and Development Study (EGDS), a novel parentoffspring adoption study with multi-informant measures of biological and adoptive parent personality and behaviour, as well as longitudinal measures of child behaviour. First, we hypothesised that observed fearlessness and low affiliative behaviour at 18 months would predict callous-unemotional behaviours at 27 months. Second, we hypothesised that biological parent fearlessness and low affiliative behaviour would predict child callous-unemotional behaviour via earlier observations of child fearlessness and low affiliative behaviour. Finally, we hypothesised that higher positive parenting of adoptive parents would protect children from heritable fearlessness and low affiliative behaviour pathways to later callous-unemotional behaviours (Fig. 2).

\section{Method}

\section{Sample}

The EGDS is a linked set of participants of 561 adopted children (42.8\% female), adoptive parents (567 adoptive mothers and 552 adoptive fathers, including 41 same-gender parents), and biological mothers $(n=554)$ and fathers $(n=208) .{ }^{23}$ On average, children were adopted within a few days of birth (median 2, range 0-91). Biological parents and children in EGDS are relatively diverse; just over half the children are White (55.6\%) and others are multiracial (19.3\%), African American (13\%) or Latino $(10.9 \%)$.

\section{Procedures}

After obtaining informed consent, questionnaires were completed via mail or the web alongside interviews and in-home assessments lasting $2-3 \mathrm{~h}$. In the current study, we used questionnaire data from adoptive mothers at child ages 9, 18, and 27 months and from biological mothers completed between 3 and 6 months postpartum. During visits to adoptive families' homes at 18 months, parents and children completed 3 min clean-up and free-play tasks (separately for adoptive mothers and fathers), a $2 \mathrm{~min}$ stranger/experimenter task, and a $1.5 \mathrm{~min}$ scary dragon task, which were recorded for observational coding. Further 
information regarding the EGDS procedures, sample, and methods is available elsewhere. ${ }^{23}$

\section{Measures}

\section{Dimensions of early child externalising}

We assessed dimensions of early externalising behaviours at 27 months using 17 items of the Achenbach System of Empirically Based Assessment. ${ }^{24}$ We formed separate callous-unemotional behaviour, oppositional behaviour, and attention deficit (ADHD) factors using a factor structure now replicated in five independent samples, including our prior work in $\mathrm{EGDS}^{10}$ (online Fig. DS1). Callous-unemotional behaviours at 27 months uniquely predicted teacher-reported externalising behaviour at age 7 within a previous EGDS report ${ }^{10}$ (online supplement DS1). By modelling other behaviour problems (i.e. oppositional and ADHD behaviours), we could test whether heritable temperament pathways were specific to callous-unemotional behaviours. We included the same five-item measure of callous-unemotional behaviours at 18 months as a covariate to account for autoregressive effects and test whether temperament predicted increases in callous-unemotional behaviours over time.

\section{Observed child fearlessness}

To assess fearlessness at 18 months, we assessed non-social behavioural inhibition (fearlessness-fearfulness) using a system based on Kochanska. ${ }^{25}$ Global coding was based on behaviour in the 2 min after the child was presented with a scary dragon toy. Fearlessness included ratings of the child approaching/touching the dragon, showing low anxiety/distress, or exhibiting disruptive behaviour. $^{25}$ Global ratings were combined into an overall fearlessness code (4-point scale; 0, shows much fearfulness/ inhibition; 4, shows fearlessness/no inhibition). A total of $15 \%$ of tapes were coded by two coders (mean intercoder agreement 92\%). Fearlessness predicted severe, chronic trajectories of antisocial behaviour from ages to 10 in a separate sample. ${ }^{26}$

\section{Observed child low affiliative behaviour}

To assess low affiliative behaviour at 18 months, we used macrosocial ratings based on interviewer global impressions of children's affiliative behaviour towards parents during free-play and clean-up tasks using items from the Coder Impressions Inventory. ${ }^{27}$ Both physically and verbally affectionate child behaviours (such as hugs, kisses, smiling at parent) were coded on a 5-point scale (1, not true/no basis; 5, multiple examples). Scores were reversed and summed across physical and verbal codes to conceptualise low affiliative behaviour. A total of $15 \%$ of tapes were coded by two independent coders (mean intercoder agreement 75\%). In support of its construct and predictive validity, lower affiliative behaviour at 18 months predicted lower teacher-reported prosocial behaviour at age 6 , over and above child gender, callous-unemotional behaviours, and other covariates (online Table DS1).

\section{Biological and adoptive mother self-report measures}

For our second hypothesis, we assessed self-reported fearlessness and low affiliative behaviours in biological mothers. Models also included these constructs for adoptive mothers to test the specificity of heritable effects.

\section{Fearlessness}

To assess fearlessness in biological and adoptive mothers, we used self-reported behavioural inhibition, based on Gray's conceptualisations of the behavioural inhibition system (BIS), a motivational system underlying approach and sensitivity to cues of punishment, non-reward, or novelty. ${ }^{28}$ We conceptualised fearlessness as low scores on the BIS (i.e. lower inhibition to negative/aversive stimuli; biological mothers $\alpha=0.73$; adoptive mothers $\alpha=0.76$ ).

\section{Affiliative behaviour}

Biological mothers completed the Harter Adult Self-Perception Profile comprising 12 four-item subscales. ${ }^{29}$ We computed the mean of three subscales that fit with conceptualisations of affiliative behaviour across different contexts and relationships with people: nurturance (for example, caring for others, particularly children, $\alpha=0.68)$, intimate relationships with a partner, spouse, or lover (for example, seeking relationships, freely communicating in relationships, $\alpha=0.74$ ), and sociability with people in general (for example, meeting new people, ease with others, $\alpha=0.79$ ). Items were rated on a forced-choice four-point scale using structured alternatives that offset the likelihood of socially desirable responses being given. Scores were coded to index low affiliative behaviour. In adoptive mothers, only 5 of these 12 subscales were collected. Thus, we computed a mean score across the nurturance $(\alpha=0.66)$ and sociability $(\alpha=0.82)$ subscales.

\section{Adoptive parent observed positive parenting}

Adoptive parents' positive parenting was assessed when children were 18 months using observed positive reinforcement during a 3 min clean-up task, with separate tasks for mothers and fathers. Videos of interactions were coded using microsocial codes derived from the Child Free Play and Compliance Task Coding Manual (K. Pears \& M. Ayers, personal communication, 2000). The frequency of positive reinforcement instances (for example 'good job', 'thanks for picking that up') were summed and a frequency proportion was calculated based on the length of the interaction. A total of $15 \%$ of tapes were coded by two independent coders (mean intercoder agreement across codes $88 \%$; overall $\kappa=0.74)$.

\section{Covariates}

Consistent with previous publications from the EGDS, we included the following covariates: child gender, degree of adoption openness (level of contact and knowledge between biological and adoptive families; see Ge et ll $^{30}$ ), and an index of perinatal risk (i.e. pre-eclampsia, prenatal substance use and low birth weight) assessed via a modification of the McNeil-Sjöström Scale for Obstetric Complications. ${ }^{31,32}$ We also controlled for adoptive mother fearlessness and low affiliative behaviour, child ADHD and oppositional behaviour at 27 months, and child callousunemotional behaviours at 18 months.

\section{Analytic strategy}

Models were tested in Mplus 7.2 using maximum likelihood procedures to account for missing data, ${ }^{33}$ and included all 561 participants (see online supplement DS1). To address the first aim, we examined associations between observed fearlessness and low affiliative behaviour and later callous-unemotional behaviours. To address the second aim, we specified a path model that tested direct effects of biological mother and adopted child fearlessness and low affiliative behaviour on later callous-unemotional behaviours and two indirect pathways: (a) biological mother fearlessness to adopted child callousunemotional behaviours via observed child fearlessness; (b) biological mother low affiliative behaviour to adopted child callous-unemotional behaviours via observed low child affiliative 
behaviour (Fig. 2). Finally, to test whether adoptive parent positive parenting moderated temperament pathways, continuous interaction terms were added to test whether adoptive mother positive parenting moderated links between fearlessness and low affiliative behaviour of biological mothers and these temperament markers for adopted children; or between child fearlessness and low affiliative behaviour and subsequent callous-unemotional behaviours (interaction terms added simultaneously). To confirm that interactions were specific to adoptive mother positive parenting, we added interaction terms to test moderation by adoptive father positive parenting (online Fig. DS2). We probed significant interactions at mean levels and 1 standard deviation above and below the mean, consistent with recommended guidelines. ${ }^{34}$

\section{Results}

Descriptive statistics and bivariate correlations between study variables are presented in online Table DS2.

\section{Are fearlessness and low affiliative behaviours related to callous-unemotional behaviour?}

We examined associations between hypothesised temperament markers and later callous-unemotional behaviours. Observations of higher fearlessness $(B=0.04$, s.e. $=0.01, P<0.01)$ and lower affiliative behaviours $(B=0.03$, s.e. $=0.01, P<0.05)$ at 18 months were related to higher adoptive mother-reported callousunemotional behaviours at 27 months, controlling for the overlap of the observed temperament measures, and also for child ADHD and oppositional behaviours, other covariates, and earlier callousunemotional behaviours (online Table DS3).

\section{Are there heritable temperament pathways to callous-unemotional behaviour?}

In a single path model, we examined whether biological mother fearlessness and low affiliative behaviour predicted child callousunemotional behaviours via earlier child temperament. In line with our hypothesis, biological mother fearlessness predicted observations of child fearlessness at 18 months $(B=0.04$, s.e. $=0.01, P<0.01)$, which in turn predicted higher callousunemotional behaviours at 27 months $(B=0.05$, s.e. $=0.02$, $\left.P<0.01 ; R^{2}=0.34\right)$. The indirect effect from biological parent fearlessness to child callous-unemotional behaviours via fearlessness was significant $(A B=0.002$, s.e. $=0.001, P<0.05$, bootstrapped $95 \%$ CI $0.003-0.050$; Fig. 3). We also found a direct effect of biological mother low affiliative behaviour on child callous-unemotional behaviours $(B=0.03$, s.e. $=0.01, P<0.01)$. In contrast to our hypothesis, biological mother and child low affiliative behaviours were unrelated, precluding testing an indirect effect on callous-unemotional behaviours.

\section{Does adoptive parent positive parenting buffer heritable temperament risk?}

To address our final aim, we re-ran the model presented in Fig. 3 and added pathways to test for continuous moderation by adoptive mother positive parenting. Adding the interaction terms

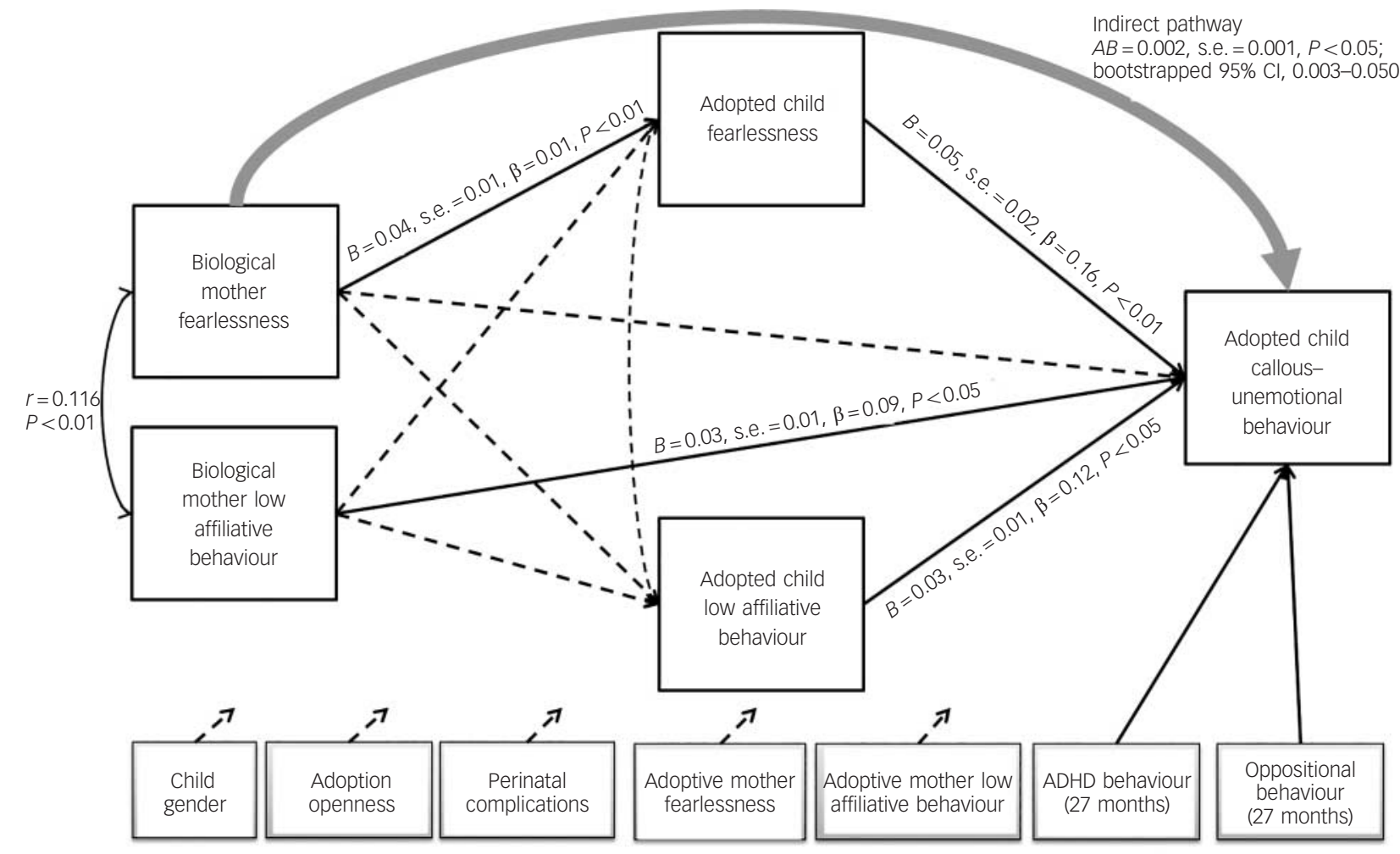

Fig. 3 Biological mother fearlessness predicts a heritable pathway to early callous-unemotional behaviour via observed child fearless temperament.

Model fit statistics: $\chi^{2}=15.09$, d.f. $=17, P>0.59$, comparative fit index $(C F I)=1.00$, root mean square error of approximation (RMSEA) $=0.00$, standardised root mean square residual $(\mathrm{SRMR})=0.02$. Callous-unemotional behaviours, $R^{2}=0.34$. Dashed lines indicate non-significant paths. We tested the indirect pathway using two methods: a product coefficient tes ('Sobel test') to quantify the magnitude of effects and unbiased confidence intervals using bootstrapping, which do not assume normality of the distribution of effects. Using maximum likelihood procedures, analyses included all participants $(n=561)$. The models controlled for the effects of the following covariates on the child callous-unemotional behaviours: child gender, adoption openness, perinatal complications, attention-deficit hyperactivity disorder (ADHD) and oppositional behaviours, and adoptive mother fearlessness and low affiliative behaviour. ADHD and oppositional behaviours were related to child callous-unemotional behaviours (Table DS1). 
significantly improved model fit $\left(\Delta \chi^{2}=67.70\right.$, d.f. $\left.=43, P<0.01\right)$ with a corresponding $2 \%$ increase in the variance in callousunemotional behaviours explained $\left(R^{2}=0.36\right)$. We found a significant interaction between adoptive mother positive parenting and child fearless temperament predicting callousunemotional behaviours $(B=-0.49$, s.e. $=0.19, P=0.01)$. We confirmed that moderation was specific to adoptive mother positive parenting by including adoptive father parenting in the model, which did not affect the pattern of significance of mother pathways, and parenting of adoptive fathers did not moderate any pathways. Positive parenting of adoptive mothers did not moderate pathways between biological mother and child fearlessness, nor between biological mother low affiliative behaviour, child low affiliative behaviour, and child callous-unemotional behaviour. In probing the significant continuous interaction of adoptive mother positive parenting with child fearlessness, we found that observed child fearlessness predicted higher callousunemotional behaviour at low $(B=0.09$, s.e. $=0.03, P<0.001)$ and mean levels $(B=0.05$, s.e. $=0.01, P<0.001)$ of adoptive mother positive parenting, but not high levels $(B=0.01$, s.e. $=0.03, P>0.69)$ (Fig. 4).

To better understand buffering of temperament risk by highly positive adoptive mothers on later callous-unemotional behaviours and compare findings within a person-centred approach, we also examined the indirect pathway using a multigroup approach. We allowed the pathway from child fearlessness to callous-unemotional behaviours that had been significant in continuous analyses to vary for adoptive mothers with high (1 s.d. $>$ mean) $v$. mean or lower levels ( $\leqslant$ mean) of positive parenting (i.e. all other pathways were fixed across groups). At high levels, the indirect pathway from biological mother fearlessness to callous-unemotional behaviours via fearlessness was not significant $(P>0.80)$. By contrast, for positive parenting at or below the mean, the indirect pathway from biological

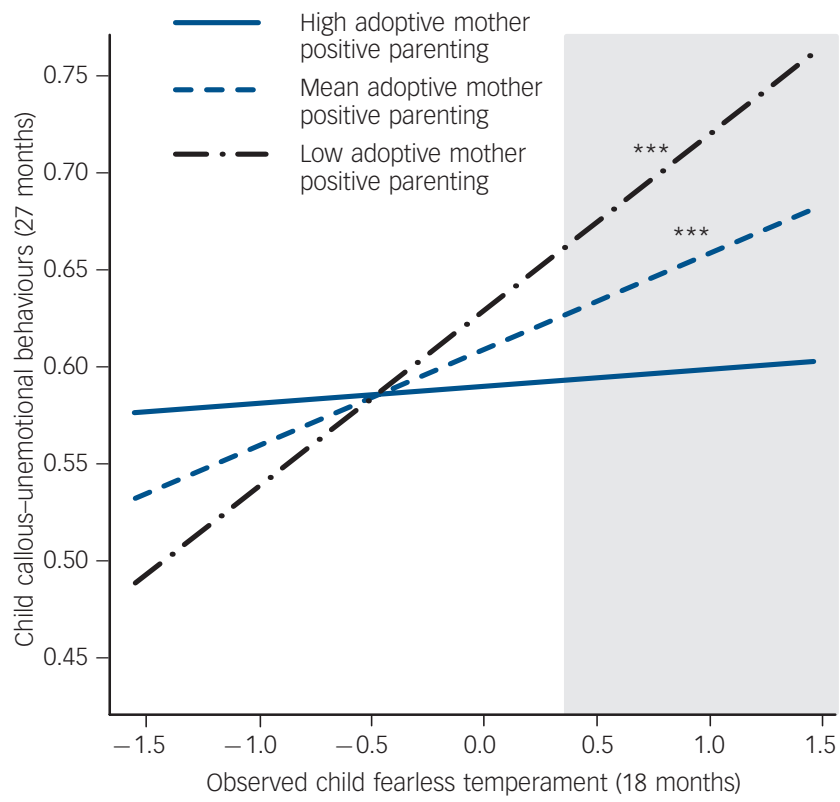

Fig. 4 High levels of adoptive mother positive parenting buffered the effect of an inherited fearless temperament on later callous-unemotional behaviour.

$* * * P<0.001$. Simple slopes plotted at mean levels, 1 s.d. above the mean, and $1 \mathrm{~s}$.d. below the mean for observed positive reinforcement, as recommended by Aiken et al..$^{35}$ Observed fearlessness in toddlerhood was related to higher

callous-unemotional behaviour at low $(B=0.09$, s.e. $=0.03, P<0.001)$ and mean levels $(B=0.05$, s.e. $=0.01, P<0.001)$ of positive reinforcement but not at high levels $(B=0.01$, s.e. $=0.03, P>0.76$ ). Region of significance indicated by grey shading (child fearless temperament scores $>0.37$ ). mother fearlessness to child callous-unemotional behaviour via fearlessness was significant $(B=0.04$, s.e. $=0.02, P<0.05)$.

\section{Discussion}

We report novel evidence of heritable temperament pathways specific to early callous-unemotional behaviours involving fearlessness and low affiliative behaviour. Biological mother fearlessness predicted adopted child callous-unemotional behaviours via earlier fearlessness, and biological mother low affiliative behaviour predicted callous-unemotional behaviours directly, although not via child affiliative behaviours. Importantly, adoptive mother observed positive parenting buffered the risk posed by early child fearlessness to later callous-unemotional behaviours. Pathways were tested in the context of an adoption study, where shared genetic influences between adoptive mother and child were eliminated and heritable $v$. non-heritable effects could be parsed. These novel findings inform developmental models of callous-unemotional behaviours and early preventive interventions.

\section{Temperament pathways \\ to early callous-unemotional behaviours}

Fearlessness is central to developmental models of callousunemotional behaviours, antisocial behaviour, and psychopathy. ${ }^{7,16,18}$ Our findings are some of the first to confirm low fear as a central developmental precursor to callous-unemotional behaviours in early childhood (also see Barker et $a^{18}$ ). Fearlessness may represent an inherited temperament marker that makes it more difficult for children to recognise and learn from signals of threat, interpersonal distress, or punishment, which increases risk for developing callous-unemotional behaviours. ${ }^{7,16}$ Our findings expand those of a recent cross-sectional study reporting a correlation between paternal psychopathic fearlessness and low child eye contact. ${ }^{19}$ This prior study was interpreted to suggest a genetic link between biological parent fearlessness and child callous-unemotional behaviours via the endophenotype of low eye contact. Our findings provide a more direct and genetically informed test of the fearlessness hypothesis through use of an adoption design and observational measures of child temperament.

In addition to fearlessness, we examined a low affiliation pathway. Biological mother and child low affiliative behaviours both predicted callous-unemotional behaviours. Because children were adopted, the direct effect of biological mother low affiliation on child callous-unemotional behaviours cannot be attributed to a history of parent-child interactions. However, we did not find evidence for an indirect heritable pathway, as biological parent and adopted child low affiliative behaviour were unrelated. This finding is surprising given the moderate heritability estimates reported in young children for constructs related to affiliation, such as empathy. ${ }^{35}$ However, one possibility to explain the null finding is that different mechanisms of heritability, including deficits in social smiling, ${ }^{36}$ could be better markers of the pathway between biological parent low affiliative behaviour and child callous-unemotional behaviours. Our brief observed measure of child verbal/physical affection may also have failed to capture individual differences in what was inherited, or was not assessed early enough in infancy relative to critical windows in the timing of parent-child attachment/affiliative behaviours. Indeed, as this measure of affiliative behaviour was observed in a dyadic context, adoptive parents may have already shaped affiliative behaviours, thus diminishing any heritable effects. Moreover, unlike the measure of fearlessness, the construct and predictive validity of 
our brief observed measure of child affiliative behaviour had not been examined in prior independent samples and may not have adequately captured the underlying construct. Nonetheless, lower scores on the measure of affiliation did directly predict increases in callous-unemotional behaviours. Thus, future work is needed to examine reciprocal associations between very early forms of social affiliation, eye contact, relationship formation with a mother or adoptive caregiver (such as social smiles or emotion response ${ }^{36}$ ), and the emergence of callous-unemotional behaviours.

\section{Moderation by parenting}

Beyond testing heritable temperament pathways, our results showed that positive parenting was particularly important for children who inherited high fearlessness. Adoptive mothers with highly positive parenting buffered the risk that fearlessness posed for the development of callous-unemotional behaviours. These findings are consistent with other studies in this sample demonstrating direct effects of adoptive mother low positive parenting on child callous-unemotional behaviours, ${ }^{10}$ and moderation of the pathway from biological mother antisocial behaviour to child callous-unemotional behaviours. ${ }^{21}$ Our present findings build on this work by testing the effects of adoptive parenting on specific temperament pathways, complementing those of other studies linking lower dyadic warmth $^{17}$ and maternal sensitivity ${ }^{37}$ to increases in callousunemotional behaviours. Thus, positive parenting strategies appear to be important in preventing the development of callous-unemotional behaviours. This message is vital when we consider heritable temperament pathways to callous-unemotional behaviours, because it emphasises that heritable does not mean unchangeable and that positive parenting is protective, even among children at high genetic and temperament risk. ${ }^{13}$

\section{Strengths and limitations}

Although our study had strengths, including an innovative design and observational measurement of child temperament and parenting, we note several limitations. First, although there was variability in child callous-unemotional behaviours, this community sample was not selected for clinical levels of antisocial behaviour, which reduces the generalisability of findings relative to forensic or clinic-referred samples. Second, we focused on adopted families, who may be less representative of the general population, given that adoptive families had more resources (income $>\$ 100000$ per year) and fewer risk factors for antisocial behaviour, whereas biological families had fewer resources and more risk for antisocial behaviour than the general population. In translating these findings, we must consider that for typical 'biological families', parents and children may share some of the same 'risky' traits, such as fearlessness and low affiliative behaviour, which interventions must contend with in order to be effective. Third, it would have been ideal to examine biological parent psychopathic traits. Unfortunately, this construct was not measured in EGDS, meaning that we could only assess temperament constructs consistent with specific traits thought to be important for callous-unemotional behaviours/psychopathy. Finally, our analyses only focused on biological mothers because data were collected from a much small number of biological fathers, precluding meaningful tests of hypothesised associations (33\% available). Thus, we likely underestimated heritability because we could not probe the extent to which fathers contributed to heritable pathways to callous-unemotional behaviours. Further, although the link between adopted child fearlessness and callous-unemotional behaviours was attenuated by adoptive mother positive parenting, there was no moderation of temperament pathways by adoptive father parenting. Given the sparse and somewhat mixed research on father behaviour in relation to the development of callous-unemotional behaviours, ${ }^{22,38}$ future research is needed to investigate the differential importance of mothers' $v$. fathers' temperament and parenting practices as predictors of callous-unemotional behaviours.

\section{Implications}

In sum, we provide compelling evidence for heritable pathways marked by fearlessness and low affiliative behaviour passed from mother to child that increase risk for callous-unemotional behaviours. Overall, our findings specify heritable temperaments for callous-unemotional behaviours that have been theorised often, but rarely tested, particularly not via an adoption design where heritable and non-heritable effects are parsed. This work demonstrates that although fearlessness and low affiliative behaviour are passed from mother to child, increasing risk for callous-unemotional behaviours, highly positive parenting can buffer risk. These results have important implications for developmental models of callous-unemotional behaviours as risk markers for pathways to antisocial behaviour.

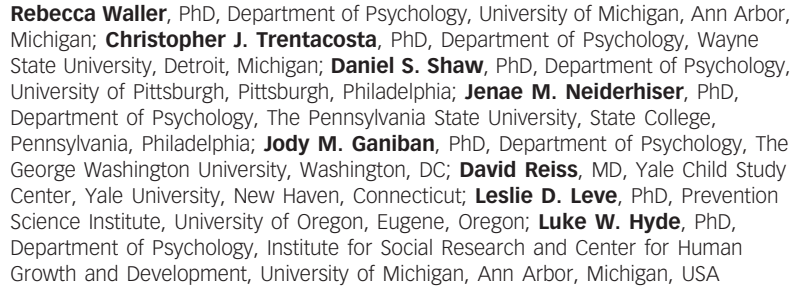

Correspondence: Luke W. Hyde, Department of Psychology, University of Michigan, 530 Church Street, Ann Arbor, MI 48109, USA. Email: lukehyde@umich.edu

First received 28 Oct 2015, final revision 15 Feb 2016, accepted 7 Apr 2016

\section{Funding}

The Early Growth and Development Study was supported by R01 and R56 HD042608 from NICHD, NIDA and OBSSR, NIH, U.S. PHS (principal investigator (PI) years 1-5: D.R.; PI years 6-10 and on the R56: L.L.); and R01 DA020585 from NIDA, NIMH and OBSSR, NIH, U.S. PHS (PI: J.N.). The content is solely the responsibility of the authors and does not necessarily represent the official views of the Eunice Kennedy Shriver National Institute of Child Health and Human Development or the National Institutes of Health. C.T. was supported in his efforts by K01 MH082926.

\section{Acknowledgements}

We thank the birth parents and adoptive families who participated in this study and the adoption agencies who helped with the recruitment of study participants. We also gratefully acknowledge Rand Conger, John Reid, Xiaojia Ge, and Laura Scaramella for their contributions to the larger project. Finally, we thank three anonymous reviewers for their helpful insights and feedback on earlier versions of the manuscript.

\section{References}

1 Foster EM, Jones DE. The high costs of aggression: public expenditures resulting from conduct disorder. Am J Public Health 2005; 95: 1767-72.

2 Shaw DS, Gross HE. What we have learned about early childhood and the development of delinquency. In The Long View of Crime: A Synthesis of Longitudinal Research (ed AM Liberman): 79-127. Springer New York, 2008.

3 Reid MJ, Webster-Stratton C, Baydar N. Halting the development of conduct problems in Head Start children: the effects of parent training. J Clin Child Adolesc Psychol 2004; 33: 279-91. 
4 Moffitt TE, Caspi A, Harrington H, Milne BJ. Males on the life-coursepersistent and adolescence-limited antisocial pathways: follow-up at age 26 years. Develop Psychopathol 2002; 14: 179-207.

5 Viding E, Fontaine NM, Mccrory EJ. Antisocial behaviour in children with and without callous-unemotional traits. J Royal Soc Med 2012; 105: 195-200.

6 McCord W, McCord J. The Psychopath: An Essay on the Criminal Mind. Van Nostrand, 1964.

7 Blair RJR. Neurobiological basis of psychopathy. Br J Psychiatry 2003; 182: 5-7.

8 Leistico AM, Salekin RT, DeCoster J, Rogers R. A large-scale meta-analysis relating the Hare measures of psychopathy to antisocial conduct. Law Hum Behav 2008; 32: 28-45.

9 Frick PJ, Ray JV, Thornton LC, Kahn RE. Can callous-unemotional traits enhance the understanding, diagnosis and treatment of serious conduct problems in children and adolescents? A comprehensive review. Psychol Bull 2014; 140: 1-57.

10 Waller R, Shaw DS, Neiderhiser JM, Ganiban JM, Natsuaki MN, Reiss D, et al. Toward an understanding of the role of the environment in the development of early callous behavior. J Personality 20 Aug 2015 (doi: 10.1111/jopy.12221)

11 Waller R, Hyde LW, Grabell AS, Alves ML, Olson SL. Differential associations of early callous-unemotional, oppositional, and ADHD behaviors: multiple domains within early starting conduct problems? J Child Psychol Psychiatry 2015; 56: 657-66.

12 Waller R, Gardner F, viding E, Shaw DS, Dishion TJ, Wilson MN, et al. Bidirectional associations between parental warmth, callous unemotional behavior and behavior problems in high-risk preschoolers. J Abnorm Child Psychol 2014; 42: 1275-85.

13 Waller R, Gardner F, Hyde LW. What are the associations between parenting callous-unemotional traits, and antisocial behavior in youth? A systematic review of evidence. Clin Psychol Rev 2013; 33: 593-608.

14 Longman T, Hawes DJ, Kohlhoff J. Callous-unemotional traits as markers for conduct problem severity in early childhood: a meta-analysis. Child Psychiatry Hum Dev 2016; 46: 327-34.

15 Rothbart MK, Ahadi SA, Evans DE. Temperament and personality: origins and outcomes. J Personal Soc Psychol 2000; 78: 122-35.

16 Kochanska G. Multiple pathways to conscience for children with different temperaments: from toddlerhood to age 5. Develop Psychol 1997; 33: 228.

17 Glenn AL, Raine A, Venables PH, Mednick SA. Early temperamental and psychophysiological precursors of adult psychopathic personality. I Abnorm Psychol 2007; 116: 508-18.

18 Barker ED, Oliver BR, Viding E, Salekin RT, Maughan B. The impact of prenatal maternal risk, fearless temperament and early parenting on adolescent callous-unemotional traits: a 14 year longitudinal investigation. J Child Psychol Psychiatry 2011; 52: 878-88.

19 Dadds MR, Allen JL, McGregor K, Woolgar M, Viding E, Scott S. Callous-unemotional traits in children and mechanisms of impaired eye contact during expressions of love: a treatment target? J Child Psychol Psychiatry 2014; 55: 771-80.

20 Vreeke G, Van der Mark I. Empathy, an integrative model. New Ideas Psychol 2003; 21: 177-207.
21 Hyde LW, Waller R, Trentacosta CJ, Shaw DS, Neiderhiser JM, Ganiban J, et al. Heritable and non-heritable pathways to early callous unemotional behavior. Am J Psychiatry 2016; April 16 (Epub ahead of print).

22 Pasalich DS, Dadds MR, Hawes DJ, Brennan J. Do callous unemotional traits moderate the relative importance of parental coercion versus warmth in child conduct problems? An observational study. J Child Psychol Psychiatry 2011; 52: 1308-15.

23 Leve LD, Neiderhiser JM, Shaw DS, Ganiban J, Natsuaki MN, Reiss D. The Early Growth and Development Study: a prospective adoption study from birth through middle childhood. Twin Res Hum Genet 2013; 16: 412-23.

24 Achenbach TM, Rescorla L. ASEBA Preschool Forms \& Profiles: An integrated System of Multi-Informant Assessment. University of Vermont Department of Psychiatry, 2000.

25 Kochanska G. Patterns of inhibition to the unfamiliar in children of normal and affectively ill mothers. Child Develop 1991; 62: 250-63.

26 Shaw DS, Lacourse E, Nagin DS. Developmental trajectories of conduct problems and hyperactivity from ages 2 to 10. J Child Psychol Psychiatry 2005; 46: 931-42.

27 Dishion T, Hogansen J, Winter C, Jabson J. Coder Impressions Inventory. 2004 (available from the Child and Family Center, University of Oregon, 195 W. 12th Avenue, Eugene, OR 97401).

28 Gray JA. A critique of Eysenck's theory of personality. In A Model for Personality (ed HJ Eysenck): 246-76. Springer Berlin Heidelberg, 1981.

29 Messer B, Harter S. Manual for the Adult Self-Perception Profile. University of Denver, 1986.

30 Ge X, Natsuaki MN, Martin DM, Leve LD, Neiderhiser JM, Shaw DS, et al. Bridging the divide: openness in adoption and postadoption psychosocial adjustment among birth and adoptive parents. J Fam Psychol 2008; 22: 529-40.

31 McNeil TF, Cantor-Graae E, Sjöström K. Obstetric complications as antecedents of schizophrenia: empirical effects of using different obstetric complication scales. J Psychiatr Res 1994; 28: 519-30.

32 Marceau K, Hajal N, Leve LD, Reiss D, Shaw DS, Ganiban JM, et al. Measurement and associations of pregnancy risk factors with genetic influences, postnatal environmental influences, and toddler behavior. Int J Behav Develop 2013; 37: 366-75.

33 Muthén LK, Muthén BO. Mplus User's Guide (7th edn) (1998-2014). Muthén \& Muthén, 2014

34 Aiken LS, West SG, Reno RR. Multiple Regression: Testing and Interpreting Interactions. Sage, 1991

35 Knafo A, Zahn-Waxler C, Van Hulle C, Robinson JL, Rhee SH. The developmental origins of a disposition toward empathy: Genetic and environmental contributions. Emotion 2008; 8: 737-52.

36 Happé F, Frith U. Annual research review: towards a developmental neuroscience of atypical social cognition. J Child Psychol Psychiatry 2014; 55: 553-77.

37 Bedford R, Pickles A, Sharp H, Wright $\mathrm{N}$, Hill J. Reduced face preference in infancy: a developmental precursor to callous-unemotional traits? Bio Psychiatry 2015; 78: 83-4.

38 Pasalich DS, Waschbusch DA, Dadds MR, Hawes DJ. Emotion socialization style in parents of children with callous-unemotional traits. Child Psychiatry Hum Dev 2014; 45: 229-42. 